\title{
A Tool to Automate Student UML diagram Evaluation
}

\author{
Salisu Modi ${ }^{1}$, Hanan A. Taher ${ }^{2}$, Hoger Mahmud ${ }^{3}$ \\ ${ }^{1}$ Department of Computer Science, Sokoto State University, Sokoto, Nigeria \\ ${ }^{2}$ Department of Information Technology Management, Duhok Polytechnic University, Duhok, Iraq \\ ${ }^{3}$ Department of Computer Science, University of Human Development, Sulaymaniyah, Kurdistan Region of Iraq
}

\begin{abstract}
Unified modelling language (UML) is the accepted standard and modelling language for modeling in software development process. UML is widely used by most course tutors in teaching modules of software engineering and system analysis and design. Students taking such courses do submit assignments with UML diagrams such as use case, class, sequence, activity and so on. Different versions of such diagrams produced by the students for a given problem have to be assessed by the course tutor which is a challenging and time-consuming task. This paper presents a java-based tool which is developed based on a simple yet effective algorithm developed by the authors that will read student and tutors solution diagrams as inputs and evaluate and grade the diagrams automatically. The output of the tool is the score of the student diagram in respect of lecturer's final solution. The output is presented in two feedback files, one containing students' score for the lecturers and the other to be send to the student to note the areas that were incorrect. The tool has been tested and evaluated using a simple and assumed UML class diagram. The result shows that the tool functions effectively and can produce detail feedbacks for both students and tutors. The outcome of this paper contributes towards automating UML diagram evaluations.
\end{abstract}

KEYWORDS: Unified Modeling Language (UML), Use case Diagram, Extensible Markup Language (XML), UML Autograding, UML diagram evaluation.

\section{Introduction}

UML stands for Unified Modeling Language. UML is a general-purpose modeling language in the field of software engineering, which is designed to provide a standard way to visualize the design of a software system. UML has become the standard for modeling object-oriented system design, ever since it was published by the object management group (OMG) [1]. UML diagrams are used to depict the architectural, dynamic, and static structures of a software system [1]. Diagrams such as class and object show the skeletal structure of classes, their interfaces, and how their objects relate to each other. On the other hand, diagrams such as activity, sequence and state machine can be used to represent the changing structure and behavior of a system [2]. Software engineers and system modelers use UML to design and develop mission critical systems in many sectors such aviation and healthcare. For examples, the authors of [3] have used UML diagrams to model and optimize hospital processes and the authors of [4] use UML to model cancer registration process. To model the dynamic nature of systems, the authors of [2] have used UML activity diagram to model service orchestration in the field of business

Teaching UML diagrams is still popular in university courses relating to software development [5]. Researchers in [6] have analyzed students' acceptance perception regarding the ease of use and usefulness of UML as a modelling tool and the outcome was positive. Positive attitude of students to use UML as modelling tool has been one of the driving factors for its wide use in software engineering courses. It is a known fact that in student-centered education environments learning by doing is essential which meaning more and more practical exercises should be given to students. Software design and development, as one of the subjects taught in higher and further education institutions, requires a lot of learning by doing exercises. In student context, diagrams usually consist of various names, nodes and connections that need to be read, compared and assessed with the solution version of the course tutor. 
Students taking such courses do submit assignment with various UML diagrams such as use cases, class, sequence, activity. Different UML model solutions can be developed by for the same problem, this has led to an issue known as "model consistency issue" [7]. Therefore, it is highly likely that diagrams developed by students in a course for a given problem are different from the diagram developed by the tutor. Because multiple solution diagrams can be created for the same problem it presents a grading challenge to instructors of these courses[8]. Grading diagrams is a time-consuming process which may take 10 to 15 minutes per student as claimed by [9]. Thus, finding a way to reduce the grading time makes a real contribution to the process of teaching UML in software design and development. To deal with the grading challenge, the authors of [8] present an approach which they call UMLGrade to streamline marking process through considering the sematic and syntactic aspects of a diagram. Following their direction, this paper presents a Java-based tool to automate the evaluation and grading of student UML diagrams. For the purpose of evaluating the tool, we use Case Study technique similar to others such as [10] [11].

The main objectives of the paper are outlined below:

- To identify a preferred UML design tool that supports all standard UML diagrams, to at least the version UML 2.2, and exports these in the XMI data format. The technique to convert models into XMI format for model evaluation purpose have been considered in other publications such as [12].

- To develop a model comparison algorithm that is able to perform strict and more relaxed comparisons between a student model and the instructor's reference model.

- To develop an assessment algorithm for students' UML diagrams that gives credit for their attempt in a way that mimics the instructor's own assessment.

- To develop a tool that can unzip a zipped file containing student diagrams and compare and assess these against the instructor's reference diagram.

- To develop a tool that can output feedbacks into readable files for both students and tutors.

The rest of this paper is organized as follows; section 2 provides some literature review on similar works, section 3 provides information on the research method, section 4 presents a brief discussion on the result and a compassion of the tool with other tools proposed in literature. Finally, in section 5 we conclude and provide some feature work directions.

\section{LITERATURE REVIEW}

The challenge of comparing and grading UML diagrams has been recognized by many researchers and there are a number of proposals in literature to deal with the challenge and automate the assessment process. The authors of [13] claim that assessment automation provides fast and consistent feedback which can be relied on by both students and teachers. To evaluate UML class diagrams, the authors of [11] use software metrics to compare the individual elements of UML class diagrams in order to identify similarities and differences between two or more diagrams. In their work, they have worked on empirically validating different software metrics with the aim to identify the most effective metrics for model comparison. The relationships between the components of class diagrams have been used by [12] to develop a method to measure similarities between two diagrams. The authors of [14] have considered the type of relations between classes to develop a calculation method for comparing student class diagrams with that of the teacher. The authors of [15] have proposed a similarity calculation method for UML class diagram based on diagram structure and semantics. 
In a similar work to what we present in this paper, the authors of [16] have presented a metamodel plus a grading algorithm to automate UML class diagram grading which maps students class diagrams to that of the instructor's in order to identify similarities and differences between them. However, they have implemented the grading algorithm in the TouchCore tool (http://touchcore.cs.mcgill.ca) but we have gone a step further to develop a tool in Java to implement our algorithm. The authors of [9] have carried out an exploratory study on the possible use of machine learning for developing automatic grading methods for UML class diagram and as a result they have concluded that machine learning is not reliable for such tasks. A UML class diagram test application have been proposed in [17] which aims to evaluate student diagrams developed for documenting an existing software. An e-assessment tool which makes use of design patterns is proposed by [18] for assessing UML class diagram.

The authors in [10] used "dynamic programming approach" to develop a mechanism to find similarities between a number of sequences diagrams with the aim to improve the efficiency of matching process. Others have used syntactic and sematic matching to evaluate Use Case diagrams [19] and the authors of [20] have proposed an architecture for assessing Use Case diagram. With improving the efficiency in software projects in mind, class property and message sequence parameters have been utilized in [21] to devise a method for comparing two sequence diagrams. The structure and semantics of sequence diagram have been considered in [15] to measure diagram similarities. The majority of these researches consider the grading or finding similarities of one type of UML diagram (e.g. class diagram), the contribution of our approach is far-reaching since our method can be applied on all types of UML diagrams. Due to the complexity of software development process and teaching the steps, automating assessments of other tasks of the development such as the programming task have also been considered by researchers. For instances, the authors of [22] have empirically investigated the automation of programming task assessment and the authors of [23] have compared two auto-assessment tools; one of the tool assess the code and the other assess the program output. This indicates that automating assessments in all areas of software development are being considered by researchers. The outcome of this paper contributes towards the effort of finding an efficient UML diagram evaluation and grading mechanism.

\section{RESEARCH METHOD}

The research method followed in this paper is comprised of four steps, step one involves identifying a UML drawing tool to be used by students and instructors to develop model solutions for a given problem which will be compatible with the Javabased tool that was developed. In step 2 the algorithm for comparing and grading student UML diagrams is developed to be implemented in the tool. In step 3 the comparison and grading tool was designed and implemented. Finally, in step 4 the tool was tested and the results were evaluated. More details about each of the steps are provided in the following subsections.

\subsection{UML Drawing Tool}

An UML drawing tool is required to be used in designing both the student version and Lecturer's solution version of a given diagram. Diagrams are designed using some standard tools, there are numerous of these tools available [13]. Different tools from different vendors comes with different specifications based on how they can handle a UML diagram. The aim of this step is to research the available UML tools, choose the most efficient for the job among them, which can be used to develop compatible models with the Java-based tool. Below are number of criteria adapted from [24] and used for selecting the best UML tool. 
- Support various diagrams: the selected tool should support the drawing of all standard UML diagrams such as class, sequence, use case, state machine and activity.

- Export/import diagram in XMI file format: the tool should be able to export diagrams in an XMI file format to be read as XML.

- Open source: the tool should allow extending and customization by intended users.

- Java code generator: the tool should generate java code equivalent of the diagram.

- Reverse engineering: the tool should be able to convert code into diagram and verse vasa.

Based on the above criteria, in table 1 four (4) UML tools (Agro UML, Modelio, Star UML, and Rational Rose) are considered and assessed (1 indicate the feature is present and 0 otherwise). Based on the assessment results both the Modelio and Star UML tools seem to have fulfilled all the criteria. Since one of is required the Moledio has been chosen to be used for designing both the student and tutor's solution version of a given diagram, which will later be read by the developed Java tool.

Table 1: Assessment of available UML drawing tools

\begin{tabular}{|c|c|c|c|c|c|c|}
\hline No. & $\frac{n}{0}$ & 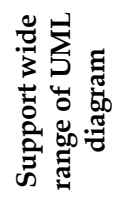 & 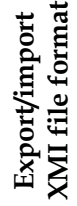 & 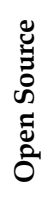 & 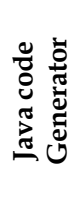 & D: \\
\hline 1 & $\begin{array}{l}\text { Agro } \\
\text { UML }\end{array}$ & 1 & 0 & 1 & 1 & 0 \\
\hline 2 & Modelio & 1 & 1 & 1 & 1 & 1 \\
\hline 3 & $\begin{array}{c}\text { Star } \\
\text { UML }\end{array}$ & 1 & 1 & 1 & 1 & 1 \\
\hline 4 & $\begin{array}{c}\text { Rational } \\
\text { Rose }\end{array}$ & 1 & 0 & 0 & 1 & 0 \\
\hline
\end{tabular}

\subsection{Comparison and Grading Algorithm}

The algorithm presented in this step is designed to compare five types of UML diagrams. The diagrams are; class, use case, activity, sequence and state machine. The algorithm designed in such a way to consider three (3) step processing. The first step is input processing. Input to the tool are two zip folders, one for students' diagrams and other for tutor solution. The diagrams are developed using Modelio tool and exported as XMI file format. A function file reader, was implemented in the tool to read the files. It then identifies type of a diagrams and read all elements in the diagram that will be compared against the same diagram type from the tutor's file. The algorithm loops until all diagrams in the folder are read. The second step is the comparison and assessment. The algorithm performs matching between elements and allocate marks to the student feedback file based on correct matches. The last step involves generating feedback file for both students and course tutor. The flowchart describes the steps of execution of the algorithm. It starts by taking in the files in XMI format. It then, reads and extracts the file using an XMI file reader. The algorithm then proceeds by picking individual element for both student diagram and tutors' diagram and compare them to identify matching elements. Marks are then allocated and feedback files are generated for both students and tutors before the algorithm terminates. Figure 1 is the pseudocode and flowchart that describe the algorithm developed.

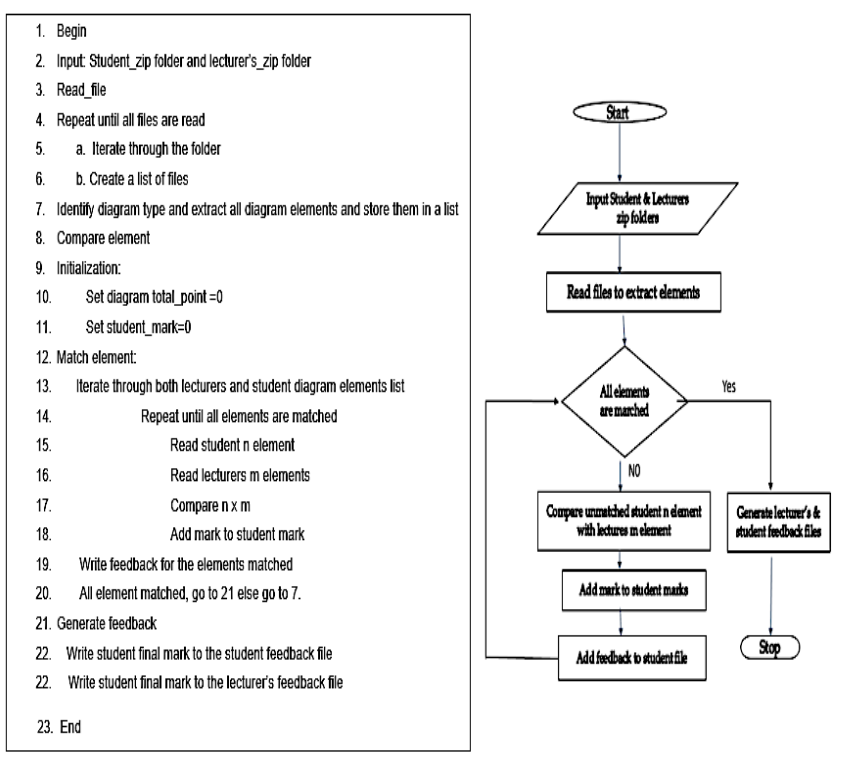

FIGURE 1: COMPARISON AND GRADING ALGORITHMS PSEUDOCODE AND FLOW CHART

\subsection{Tool Design and Implementation}

The overall tool implementation is subdivided into 
four (4) logical units as shown in figure 2. The first component handles how input to the tool is being processed. The submission has to be in zip format containing all students' assignment, then the content of files are read and individual elements are extracted. The second subcomponent identifies each element for the comparison process. The third component compares individual student diagram element against solution diagram and allocate marks. The last component generate feedback to both student and course tutor.

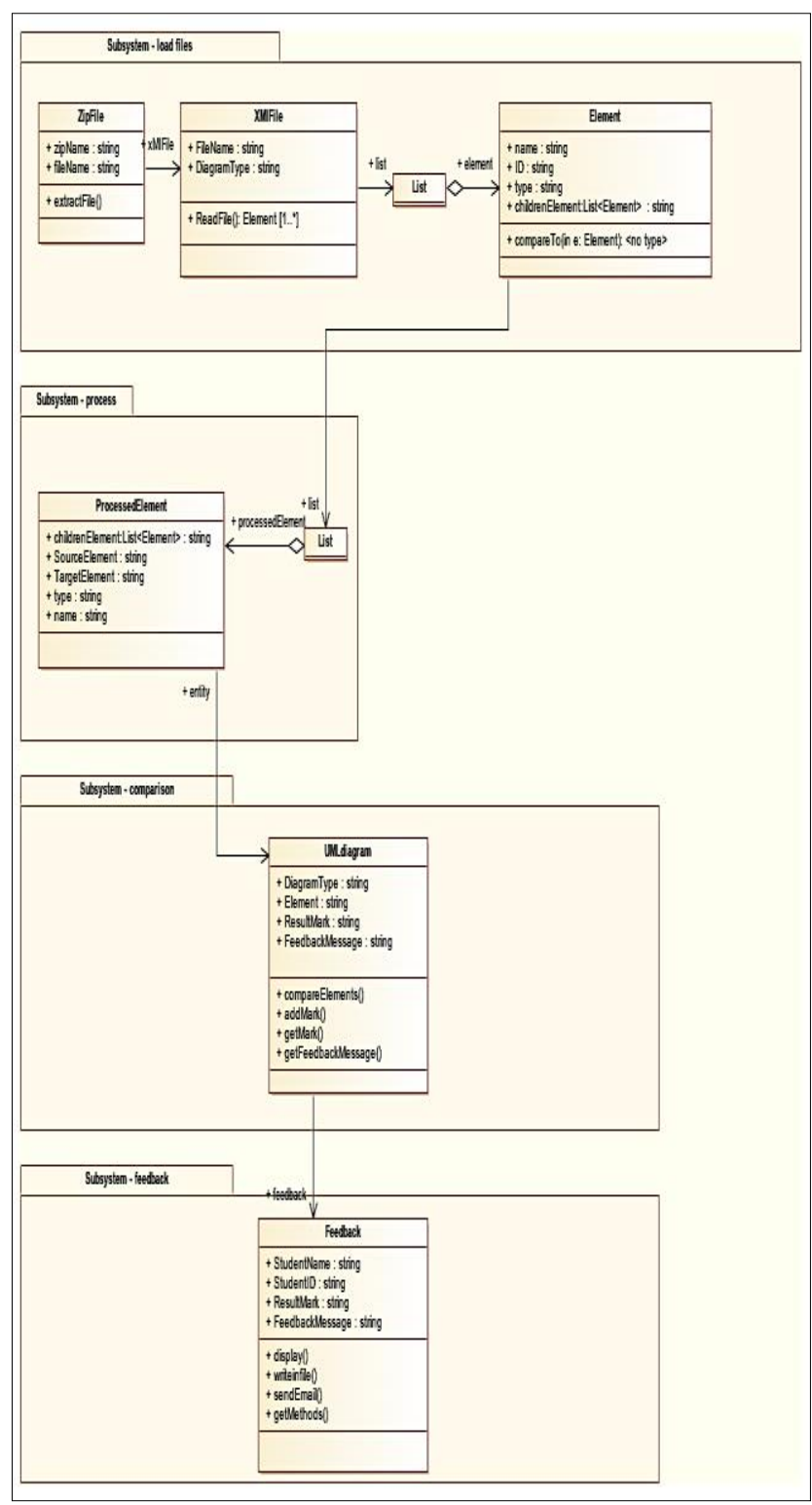

FIGURE 2: THE UML COMPARISON AND GRADING TOOL COMPONENTS DESIGN

The tool is intended to be a standalone, as it will be used by the course tutor for assessing students' diagrams. The interface of the tool allows the user (tutor) to interact with the tool in such a way that he/she uploads his/her own version of UML diagram in XMI format, then upload the student's UML diagram which is also in XMI format. After uploading both files, the user will click the "compare button" which will start the comparison process. There is a processing bar showing the approximate completion percentage of the comparison process. After completion, the result is displayed in the display pan and a copy is of the result saved to a file which will be send as a feedback. Figure 3 shows the home interface of the tool developed in Java.

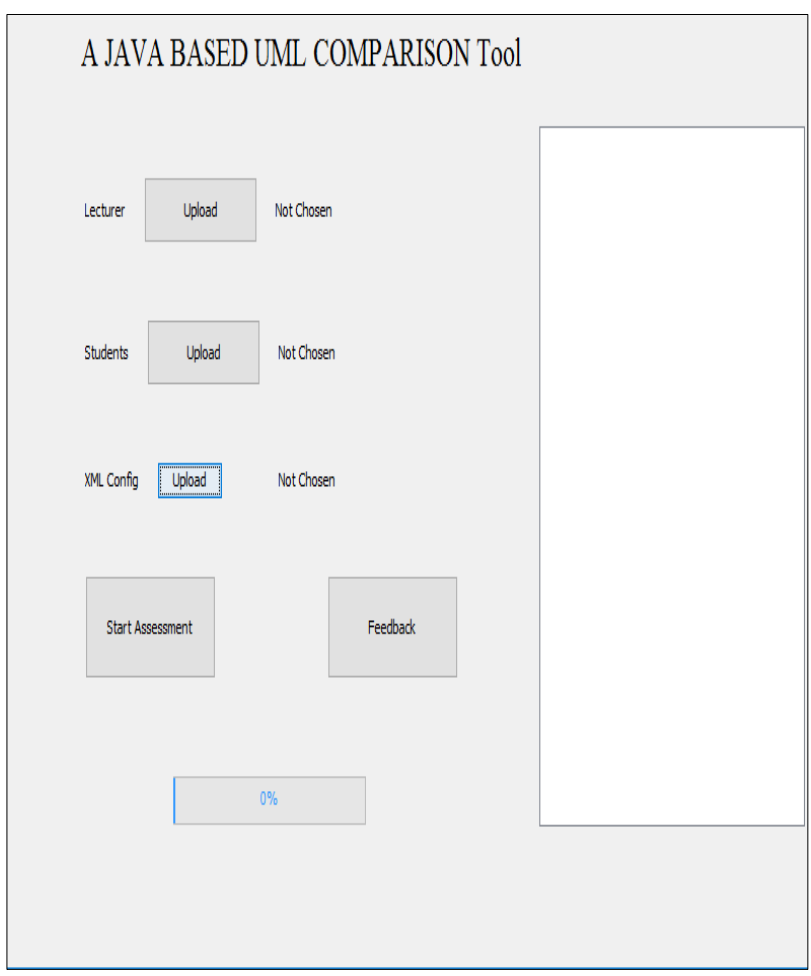

FIGURE 3: THE COMPARISON AND GRADING TOOL HOME INTERFACE

\subsection{Tool test and Evaluation}

To test the functionality of the tool, a class diagram example is assumed and drawn for the tutors to be used as a comparison base against the possible student solution. The process is the same for all other types of UML diagrams, therefore, it was deemed not necessary to provide examples of other types of diagrams. Both the tutors and possible student diagrams are described in the sections 3.4.1 and 3.4.2 and test results is presented in section 3.4.3 in a form of student and tutor feedback. 


\subsubsection{Tutor's Diagram}

The tutor's solution diagram is shown in figure 4 . The diagram consists of six (6) classes including two (2) association classes. The association classes are between class1 and class 2 as well as between class 4 and class5. The class diagram is developed with all possible features of a typical diagram in mid such as attributes, operations and associations in order to see how the tool copes with the features; also, to ensure that the tool can handle all possible class diagram features that students and teachers may include in their solution diagrams.

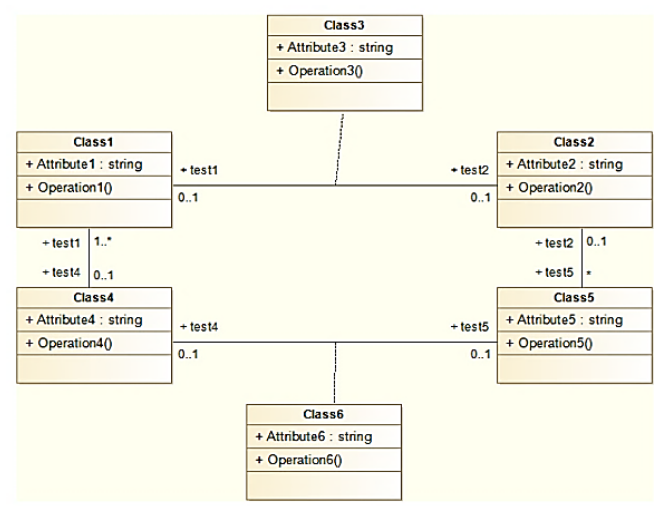

Figure 4: Tutor's Solution Class Diagram

\subsubsection{Students Diagrams}

The diagrams in figure 5 and Figure 6 are two possible solution diagrams assumed to have been submitted by students. The diagram in figure 5 presents student with submission No. aca12asa diagram. The diagram consists of six (6) classes as in the tutor's diagram, but association name (value) for class 1 and class 2 in tutors' diagram was test 1 and test 2 while in that of the student was test 11 and test 22 , therefore the student loses some marks. Similarly, figure 6 presents student 2 with submission No. aca13asa diagram. Although the diagram is comprised of the same number of classes as in tutor's diagram, but the association class between class 4 and class 5 is wrongly placed in the student diagram between class 2 and class5, therefore student 2 also loses some marks for the association class. Figure 7 shows both students and tutors sample solutions are uploaded and processed successfully. The result of comparison sent to the students as feedback is shown in table 2

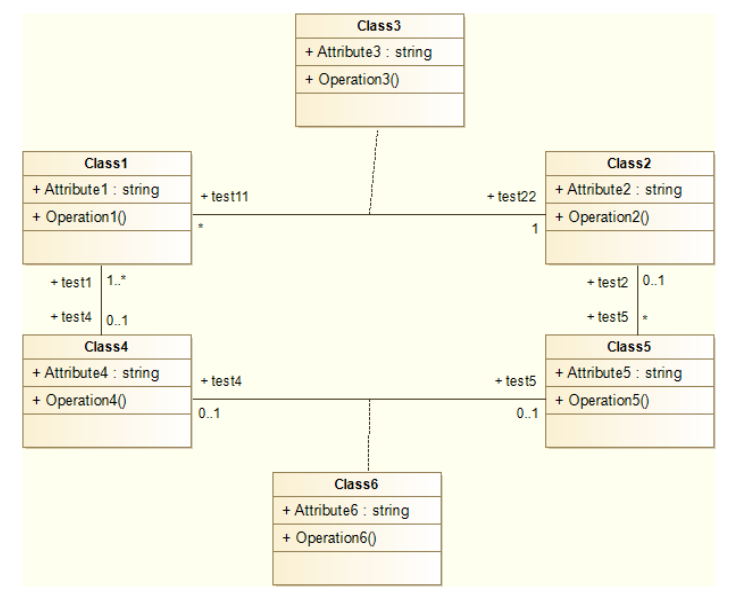

Figure 5: Student1 (aca13asa) different association values from that of the tutor's diagram

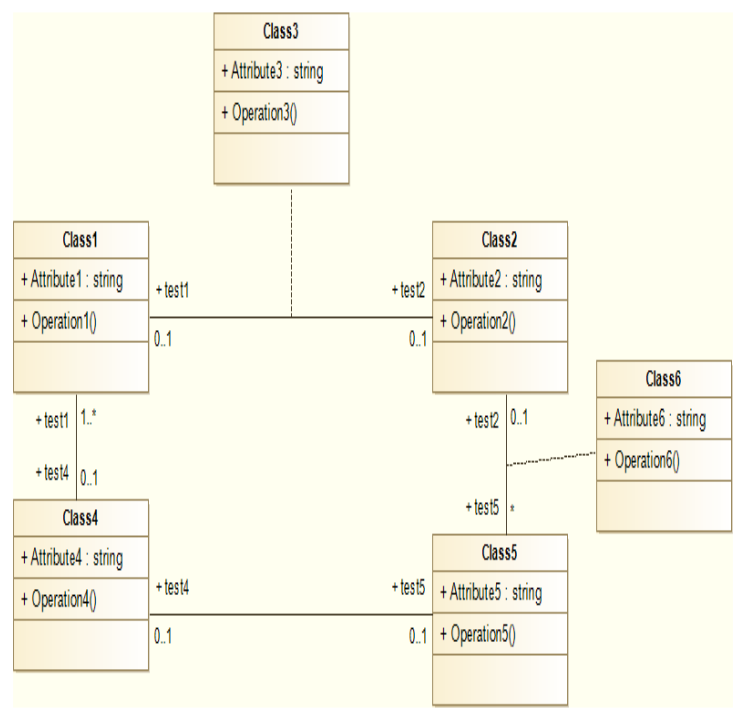

Figure 6: Student2 (aca12asa) different positioning of association class from that of the tutor's diagram

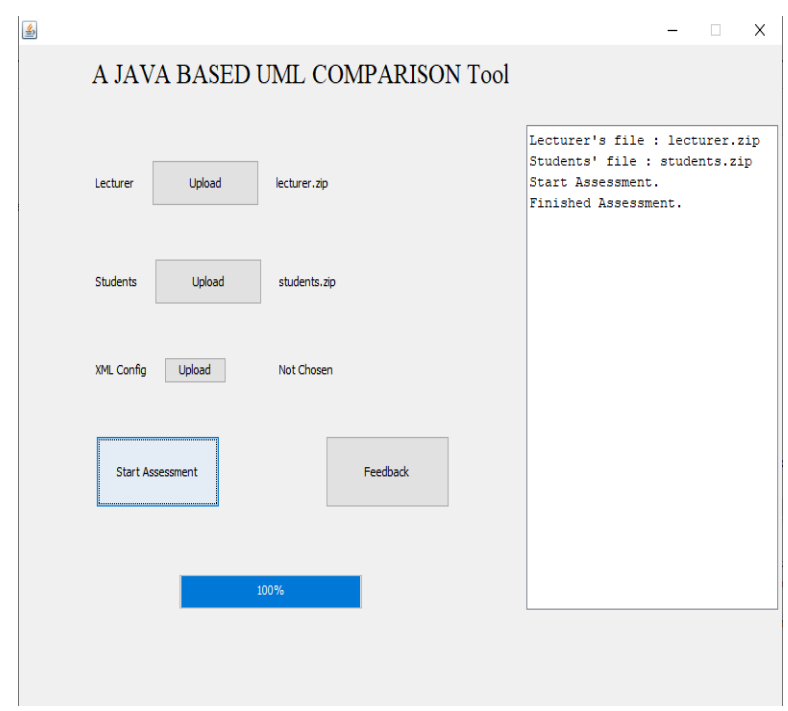

Figure 7: Sample test-running of the tool with tutor's and students diagram above

\subsubsection{Feedback}


The tool produces two forms of feedback files which are student and tutor's file. The students feedback contains student information, marked scored on each diagram and information about the comparison showing the student the elements of the diagram that are correct and the once that are not correct. Table 2 is the description of the content of a sample student feedback file. The tutor's feedback file contains the list of students' information and the marked scores for each diagram. Figure 8 is a sample feedback file for the tutor.

Table 2: Sample content of student feedback file for the class diagrams (in figure 5 and 6) assessment.

\begin{tabular}{|c|c|c|c|}
\hline & $\begin{array}{c}\text { Diagram } \\
\text { type }\end{array}$ & $\begin{array}{c}\text { Marked } \\
\text { scored }\end{array}$ & Feedback info. \\
\hline $\begin{array}{l}\text { Id: aca13asa } \\
\text { Name: } \\
\text { Abdulrahem } \\
\text { Al Abri }\end{array}$ & $\begin{array}{l}\text { Class } \\
\text { diagram }\end{array}$ & 67 & $\begin{array}{l}\text { Uml element type: Class } \\
\text { Class1- element is correct } \\
\text { Uml element type: Class } \\
\text { Class2- element is correct } \\
\text { Uml element type: Class } \\
\text { Class4- element is correct } \\
\text { Uml element type: Class } \\
\text { Class5- element is correct } \\
\text { Uml element type: } \\
\text { Association } \\
\text { first member:=- } \\
\text { Class4:second member- } \\
\text { Class1 } \\
\text { element is correct } \\
\text { Uml element type: } \\
\text { Association } \\
\text { first member:=- } \\
\text { Class5:second member- } \\
\text { Class2 } \\
\text { element is missing } \\
\text { Uml element type: } \\
\text { AssociationClass } \\
\text { Class3-element is correct } \\
\text { AssociationClass } \\
\text { Class6- element is missing }\end{array}$ \\
\hline $\begin{array}{l}\text { Id: aca12asa } \\
\text { Name: } \\
\text { Abdulrahem } \\
\text { Al Abri }\end{array}$ & $\begin{array}{l}\text { Class } \\
\text { diagram }\end{array}$ & 91 & $\begin{array}{l}\text { Uml element type: Class } \\
\text { Class1- element is correct } \\
\text { Uml element type: Class } \\
\text { Class2- element is correct } \\
\text { Uml element type: Class } \\
\text { Class4- element is correct } \\
\text { Uml element type: Class } \\
\text { Class5- element is correct } \\
\text { Uml element type: } \\
\text { Association }\end{array}$ \\
\hline
\end{tabular}

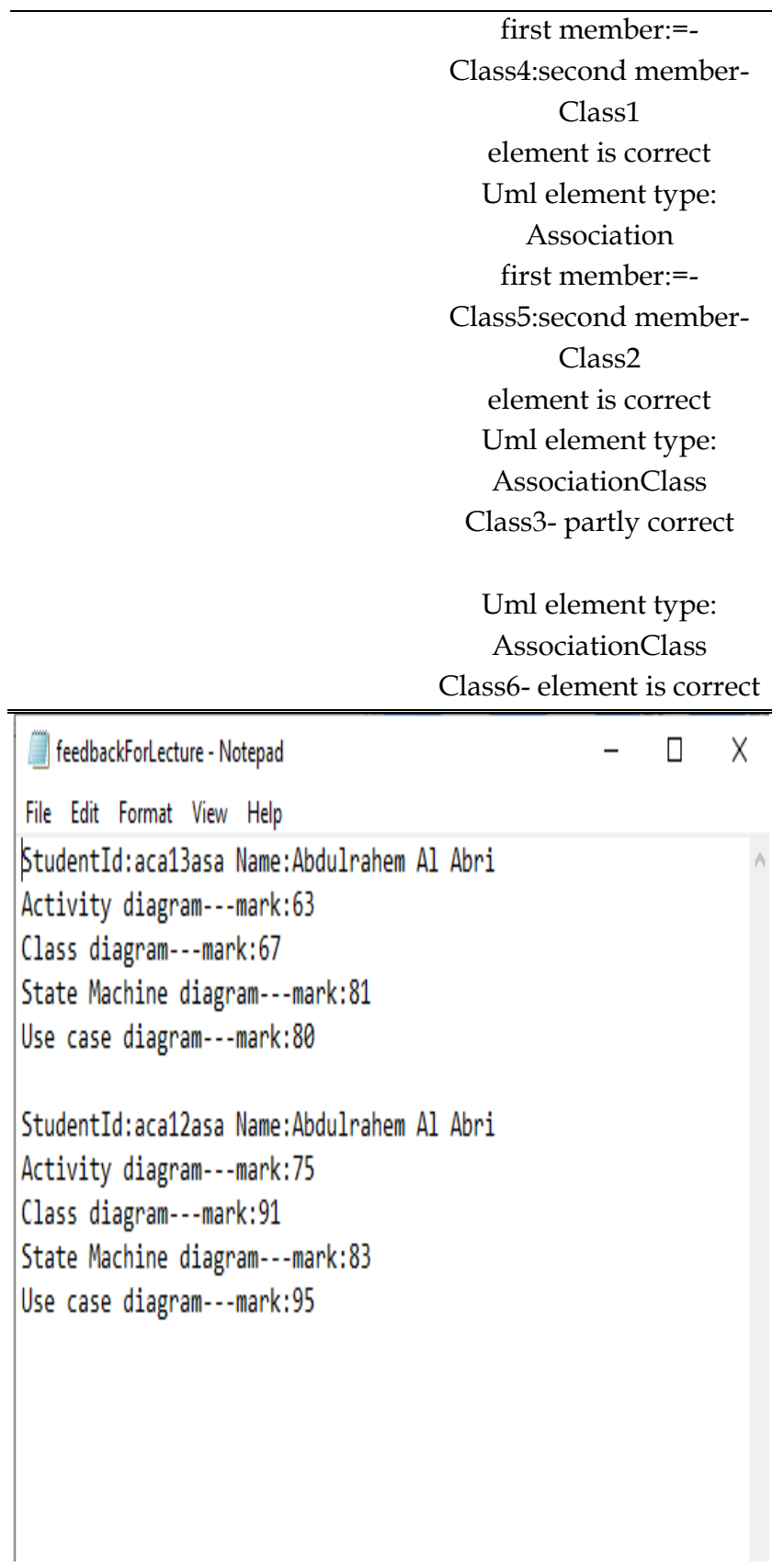

Figure 8: The content of a sample feedback file for the lecturer.

\section{DISCUSSION}

It is claimed by [18] that e-assessment is taking a foothold in higher and further education institutions despite its complexity in implementation. For instance, the Open University is expanding the use of its online quiz engines with the aim to facilitate the process of marking and providing feedback to its students [25]. In this paper, we have introduced a student UML evaluation tool which grades student's by comparing their submitted solution to that of the tutor's. The initial version of the tool is specialized to 
just diagrams created by Modeli. Ideally, the tool should handle diagrams created by any modeling tools which is something we intend to work on in the future. In the proposed tool we use feature matching technique to match diagrams between students and tutor answers, which is a qualitative approach for diagram matching. This approach is different from others such as [10] which has used a quantitative approach by calculating Mean Average Precision to decide on matching quality between two more diagrams. To showcase the difference in tool development approach table 3 provides a comparison of the developed tool with five other proposed tools in literature [16] [17] [12][26] [27] . The comparison is carried out based on the following criteria:

- The utilization of a standard UML tool: this criterion examines the tools in terms of the use of standard UML diagram creation tool.

- XMI export: this criterion examines the tools in terms of the ability to export produced diagrams in XMI format which facilitates the diagram comparison process in the developed tool.

- Multi-assessment capability: this criterion examines the ability of the tools in terms of assessing multiple types of UML diagrams.

- File type input: this criterion examines the ability of the tools in terms of accepting single or multiple files.

- Auto-grading: this criterion examines the ability of the tools in terms of automatically grading student diagrams.

- Generation of feedback file: this criterion examines the ability of the tools to generate student and tutor feedback files.

The comparison results presented in table 3 shows that the Auto-UML evaluation tool developed in this paper is more advanced than the proposed tools in literature based on the specified criteria. Finally, this leads us to claim that the developed tool can facilitate the automation of UML diagrams evaluation more comprehensively that the proposed tools in literature. It is clear that this kind of tools can assist instructors in dealing with student diagram evaluation and grading challenges by reducing time and effort in the process. One other use of UML diagram comparison or matching tool is to reuse past UML design diagrams that have been implemented in developing existing software tools [10] [21]. This is particularly useful when new functionalities are going to be added to an existing tool which reduces design and development time and cost.

Table 3: The comparison of the developed tool to other similar tools presented in literature

No.

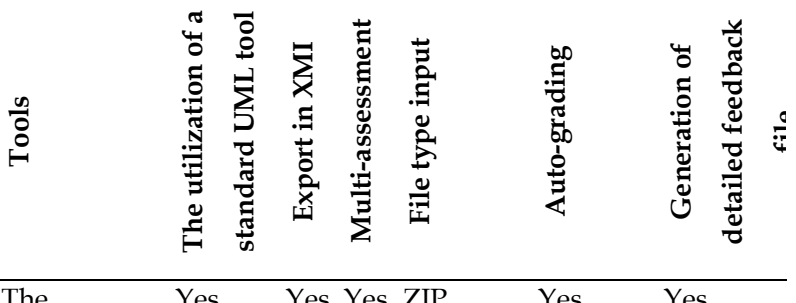

developed (Multi-file

UML tool in input

this work capability)

\begin{tabular}{lll}
\hline $\begin{array}{l}\text { Automated No } \\
\text { Grading of }\end{array}$ & No No Sigle plain Yes & Yes/ \\
text file & feedback \\
class diagram & & is only \\
{$[16]$} & & produced \\
& & for \\
& & student
\end{tabular}

3 UML test Yes Yes No A single Partially, yes application UXF file evaluates for diagram automated but no validation of mark UML class allocation diagram [17]

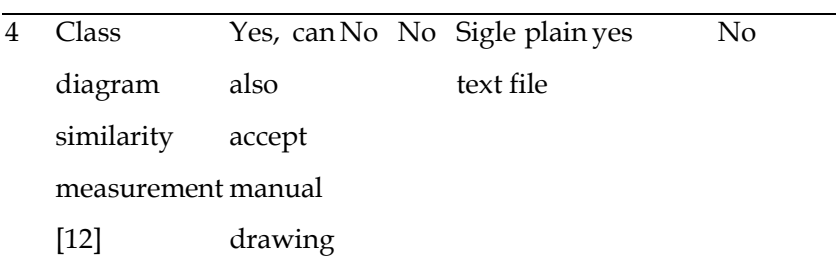




\begin{tabular}{llll}
\hline 5 Mistakes in No No Yes Yes & $\begin{array}{l}\text { No, No } \\
\text { generates }\end{array}$ \\
UML & & a \\
diagrams: & & catalogue \\
analysis of & & of \\
student & & diagram & mistakes \\
projects in a & & & \\
software & & Yes & Yes \\
engineering & & & \\
course [26] & & & \\
Automated Yes & Yes No Single \\
Checks on & XMI file & \\
UML & & \\
diagrams & & \\
[27] & & \\
\hline \hline
\end{tabular}

\section{CONCLUSION AND FUTURE WORK}

Tutors teaching software engineering and system analysis and design courses suffer tremendously from manually assessing student UML diagrams. In this paper we have presented the design and implementation of an automated java-based tool for comparing and assessing students UML diagrams against tutor solution diagrams. The tool takes in two zip files, as in put, containing diagrams in XMI format for both students and the tutor which are created using Modellio UML tool. The tool generates two feedback files, one for the students containing student marks and some guideline information regarding the assessed diagram and the other for the tutor containing all the student information and their final mark for each diagram. The objective of this research is to provide an alternative mean to manual assessment of student UML diagrams in this digital era.

The current tool is a desktop application, in the future we intend to develop a web-based application version of the tool. This is to facilitate the comparison and grading process by enabling students to upload their diagrams and get immediate feedback without the need to wait for tutors to send them the feedback.

\section{REFERENCES}

1. P. Kumarapeli, S. De Lusignan, T. Ellis, and B. Jones, "Using Unified Modelling Language (UML) as a process-modelling technique for clinical-research process improvement," Informatics for Health and Social Care, vol. 32, no. 1, pp. 51-64, 2007.

2. P. Mayer, A. Schroeder, and N. Koch, "MDD4SOA: Model-driven service orchestration," in Enterprise Distributed Object Computing Conference, 2008. EDOC'08. 12th International IEEE, 2008, pp. 203-212.

3. G. T. Jun, J. Ward, Z. Morris, and J. Clarkson, "Health care process modelling: which method when?," International Journal for Quality in Health Care, p. mzp016, 2009.

4. S. Garde, B. Baumgarten, O. Basu, N. Graf, R. Haux, R. Herold, U. Kutscha, F. Schilling, B. Selle, C. Spiess, and others, "A meta-model of chemotherapy planning in the multi-hospital/multi-trial-center-environment of pediatric oncology," Methods Inf Med, vol. 43, no. 2, pp. 171-183, 2004.

5. A. M. Fernández-Sáez, M. R. Chaudron, and M. Genero, "An industrial case study on the use of UML in software maintenance and its perceived benefits and hurdles," Empirical Software Engineering, vol. 23, no. 6, pp. 3281-3345, 2018.

6. A. Lopes, I. Steinmacher, and T. Conte, "UML Acceptance: Analyzing the Students' Perception of UML Diagrams," in Proceedings of the XXXIII Brazilian Symposium on Software Engineering, 2019, pp. 264-272.

7. F. J. Lucas, F. Molina, and A. Toval, "A systematic review of UML model consistency management," Information and Software Technology, vol. 51, no. 12, pp. 1631-1645, 2009.

8. K. Farias and B. C. da Silva, "What's the grade of your diagram? towards a streamlined approach for grading UML diagrams," in Proceedings of the 23rd ACM/IEEE International Conference on Model Driven Engineering Languages and Systems: Companion Proceedings, 2020, pp. 1-2.

9. D. R. Stikkolorum, P. van der Putten, C. Sperandio, and M. Chaudron, "Towards Automated Grading of UML Class Diagrams with Machine Learning.," in BNAIC/BENELEARN, 2019.

10. A. Adamu and W. M. N. W. Zainon, "Similarity Assessment of UML Sequence Diagrams Using Dynamic Programming," in International Visual Informatics Conference, 2017, pp. 270-278.

11. M. A.-R. Al-Khiaty and M. Ahmed, "UML class diagrams: Similarity aspects and matching," Lecture Notes on Software Engineering, vol. 4, no. 1, p. 41, 2016.

12. R. Fauzan, D. Siahaan, S. Rochimah, and E. Triandini, “Class Diagram Similarity Measurement: A Different Approach," in 2018 3rd International Conference on Information Technology, Information System and Electrical Engineering (ICITISEE), 2018, pp. 215-219.

13. C. K. Poon, T.-L. Wong, C. M. Tang, J. K. L. Li, Y. T. Yu, and V. C. S. Lee, "Automatic assessment via intelligent analysis of students' program output patterns," in International Conference on Blended Learning, 2018, pp. 238-250. 
14. M. A.-R. Al-Khiaty and M. Ahmed, "Matching UML class diagrams using a Hybridized Greedy-Genetic algorithm," in 2017 12th International Scientific and Technical Conference on Computer Sciences and Information Technologies (CSIT), 2017, vol. 1, pp. 161-166.

15. D. Siahaan, Y. Desnelita, and others, "Structural and semantic similarity measurement of UML sequence diagrams," in 2017 11th International Conference on Information IE Communication Technology and System (ICTS), 2017, pp. 227-234.

16. W. Bian, O. Alam, and J. Kienzle, "Automated grading of class diagrams," in 2019 ACM/IEEE 22nd International Conference on Model Driven Engineering Languages and Systems Companion (MODELS-C), 2019, pp. 700-709.

17. P. Herout and P. Brada, "UML-Test Application for Automated Validation of Students' UML Class Diagram," in 2016 IEEE 29th International Conference on Software Engineering Education and Training (CSEET), 2016, pp. 222-226.

18. T. Reischmann and H. Kuchen, "Towards an Eassessment tool for advanced software engineering skills," in Proceedings of the 16th Koli Calling International Conference on Computing Education Research, 2016, pp. 81-90.

19. V. Vachharajani and J. Pareek, "Framework to approximate label matching for automatic assessment of use-case diagram," International Journal of Distance Education Technologies (IJDET), vol. 17, no. 3, pp. 75-95, 2019.

20. V. Vachharajani and J. Pareek, "A proposed architecture for automated assessment of use case diagrams," International Journal of Computer Applications, vol. 108, no. 4, 2014.

21. E. Triandini, R. Fauzan, D. O. Siahaan, and S. Rochimah, "Sequence diagram similarity measurement: a different approach," in 2019 16th International Joint Conference on Computer Science and Software Engineering (JCSSE), 2019, pp. 348-351.

22. J. Liebenberg and V. Pieterse, "Investigating the feasibility of automatic assessment of programming tasks," 2018.

23. A. Bey, P. Jermann, and P. Dillenbourg, "A comparison between two automatic assessment approaches for programming: An empirical study on MOOCs," Journal of Educational Technology $\mid \mathcal{E}$ Society, vol. 21, no. 2, pp. 259-272, 2018.

24. T. Rani and S. Garg, "Comparison of different UML tool: Tool approach," International Journal Of Engineering And Computer Science, vol. 2, no. 6, pp. 1900-1908, 2013.

25. P. Thomas, "Online Automatic Marking of Diagram," Springer, vol. 26, no. 349-359, 2012.

26. S. Chren, B. Buhnova, M. Macak, L. Daubner, and B. Rossi, "Mistakes in UML diagrams: analysis of student projects in a software engineering course," in 2019 IEEE/ACM 41st International Conference on Software Engineering: Software Engineering Education and Training
(ICSE-SEET), 2019, pp. 100-109.

27. M. Striewe and M. Goedicke, "Automated checks on UML diagrams," in Proceedings of the 16th annual joint conference on Innovation and technology in computer science education, 2011, pp. 38-42. 